EUROPEAN JOURNAL OF PURE AND APPLIED MATHEMATICS

Vol. 12, No. 1, 2019, 146-158

ISSN 1307-5543 - www.ejpam.com

Published by New York Business Global

\title{
On a Graph Induced by a Hyper BCI-algebra
}

\author{
Michelle T. Panganduyon ${ }^{1}$, Sergio R. Canoy, Jr. ${ }^{1, *}$ \\ Department of Mathematics and Statistics, College of Science and Mathematics, \\ Center for Graph Theory, Algebra, and Analysis-PRISM, Mindanao State University-Iligan \\ Institute of Technology, 9200 Iligan City, Philippines
}

\begin{abstract}
This paper introduces the notion of the zero divisor graph of a hyper BCI-algebra and investigates some of its properties.
\end{abstract}

2010 Mathematics Subject Classifications: 20M14, 05C25

Key Words and Phrases: Zero divisor graph, hyper BCI-algebra, hyperatom, complete, star

\section{Introduction}

Graph Theory and Abstract Algebra have been profoundly studied by mathematicians because of the interesting topics laid upon these branches of mathematics. Indeed, some authors studied graph theory to build connections with certain algebraic structures such as commutative semigroups, commutative rings, and non-commutative rings. Beck, in his work in [1], associated to any commutative ring $R$ its zero divisor graph $G(R)$ whose vertices are the zero divisors of $R$ (including an element 0 of $R$ ) and where adjacency between two distinct elements of $R$ is defined as follows: two vertices $x, y$ are adjacent if and only if $x y=0$. In 2002, DeMeyer et al. [2] also pioneered the notion of zero-divisor graph of commutative semigroup $S$ with 0 . They associated an undirected graph $\Gamma(S)$ to any commutative semigroup $S$ with 0 whose vertices are the nonzero zero divisors of $S$, such that two vertices $x, y$ are adjacent if and only if $x y=0$. More recently, Y. B. Jun and K. J. Lee [5] introduced the concept of associated graph of BCI-algebra and verified some properties of the graph. Motivated by these works, in this paper, we shall introduce the notion of the zero divisor graph of a hyper BCI-algebra and investigate some of its properties.

* Corresponding author.

DOI: https://doi.org/10.29020/nybg.ejpam.v12i1.3362

Email addresses: michelle.panganduyon@g.msuiit.edu.ph (M. Panganduyon), sergio.canoy@g.msuitt.edu.ph (S. Canoy) 


\section{Preliminaries}

The concepts on Graph Theory are taken from [4]:

A graph $G$ is an ordered pair $(V(G), E(G))$, where $V(G)$ is a finite nonempty set called the vertex set of $G$ and $E(G)$ is a set of unordered pairs $\{u, v\}$ (or simply $u v$ ) of distinct elements from $V(G)$ called the edge set of $G$. The elements of $V(G)$ are called vertices and the cardinality $|V(G)|$ of $V(G)$ is the order of $G$. The elements of $E(G)$ are called edges and the cardinality $|E(G)|$ of $E(G)$ is the size of $G$. A graph $K=(V(K), E(K))$ is a subgraph of a graph $G=(V(G), E(G))$ if $V(K) \subseteq V(G)$ and $E(K) \subseteq E(G)$. Two vertices $u, v$ of a graph $G$ are adjacent, or neighbors, if $u v$ is an edge of $G$. The set of neighbors of a vertex $v$ of $G$ is denoted by $N_{G}(v)$ and the degree of $v$ in $G$, denoted $\operatorname{deg} v$, is equal to $\left|N_{G}(v)\right|$. The degree of $G$, denoted by $\Delta(G)$, is equal to the largest degree of a vertex of $G$. A vertex $w$ of $G$ is called an isolated vertex if $\operatorname{deg}_{G}(w)=\left|N_{G}(w)\right|=0$. The set of all isolated vertices of $G$ will be denoted by $\mathcal{I}(G)$. A graph $G$ is called an empty graph, denoted by $\bar{K}_{|V(G)|}$, if $E(G)=\varnothing$, that is, $\mathcal{I}(G)=V(G)$.

A walk of a graph $G$ is an alternating sequence of vertices and edges, beginning and ending with vertices, $v_{0}, e_{1}, v_{1}, \ldots, v_{n-1}, e_{n}, v_{n}$, in which each edge is incident with the two vertices immediately preceding and following it. This walk joins $v_{0}$ and $v_{n}$; and is sometimes called a $v_{0}-v_{n}$ walk. It is closed if $v_{0}=v_{n}$ and is open otherwise. It is a path if all the vertices (and thus necessarily all the edges) are distinct. If the walk is closed, then it is a cycle provided its $n$ vertices are distinct and $n \geq 3$. We denote by $C_{n}$ the graph consisting of a cycle with $n$ vertices and by $P_{n}$ a path with $n$ vertices.

A graph is connected if every pair of vertices are joined by a path. A maximal connected subgraph of $G$ is called a component of $G$. The complete graph $K_{p}$ has every pair of its $p$ vertices adjacent. A bipartite graph $G$ is a graph whose vertex set $V$ can be partitioned into two subsets $V_{1}$ and $V_{2}$ such that every edge of $G$ joins $V_{1}$ with $V_{2}$. If $G$ contains every edge joining $V_{1}$ and $V_{2}$, then $G$ is a complete bipartite. If $V_{1}$ and $V_{2}$ have $m$ and $n$ vertices, respectively, then we write $G=K_{m, n}$. A star is a complete bipartite $K_{1, n}$.

The Kronecker product $G \otimes K$ of two graphs $G$ and $K$ is the graph with vertex set $V(G \otimes K)=V(G) \times V(K)$ and edge set $E(G \otimes K)$ satisfying the following conditions: $(x, u)(y, v) \in E(G \otimes K)$ if and only if $x y \in E(G)$ and $u v \in E(K)$.

Let $G$ and $K$ be graphs and let $f: V(G) \rightarrow V(K)$ be a function. Then $f$ is a graph homomorphism if $f(x) f(y) \in E(K)$ whenever $x y \in E(G)$. Two graphs $G$ and $K$ are isomorphic (written as $G \cong K$ ) if there exists a one-to-one correspondence between the vertex sets which preserves adjacency.

A hyperoperation on a nonempty set $H$ is a map from $H \times H$ into $P^{*}(H)=P(H) \backslash\{\varnothing\}$. Let $\circledast$ be a hyperoperation on $H$ and $(x, y) \in H \times H$. Then its image under $\circledast$, denoted by $x \circledast y$, is called the hyperproduct of $x$ and $y$. If $A$ and $B$ are nonempty subsets of $H$, then $A \circledast B$ is given by $A \circledast B=\bigcup_{a \in A, b \in B} a \circledast b$. We shall use $x \circledast y$ instead of $x \circledast\{y\},\{x\} \circledast y$, or $\{x\} \circledast\{y\}$. When $A \subseteq H$ and $x \in H$, we agree to write $A \circledast x$ instead of $A \circledast\{x\}$. Similarly, we write $x \circledast A$ for $\{x\} \circledast A$. In effect, $A \circledast x=\bigcup_{a \in A} a \circledast x$ and $x \circledast A=\bigcup_{a \in A} x \circledast a$. 
A hyper $B C I$-algebra $(H, \circledast, 0)$ is a nonempty set $H$ endowed with a hyperoperation " $\circledast$ " and a constant 0 satisfying the following axioms: for all $x, y, z \in H$,

$\left(B_{1}\right)((x \circledast z) \circledast(y \circledast z)) \ll x \circledast y$,

$\left(B_{2}\right)(x \circledast y) \circledast z=(x \circledast z) \circledast y$,

$\left(B_{3}\right) x \ll x$,

$\left(B_{4}\right) x \ll y$ and $y \ll x$ imply $x=y$,

$\left(B_{5}\right) 0 \circledast(0 \circledast x) \ll x, x \neq 0$,

where for every $A, B \subseteq H, A \ll B$ if and only if for each $a \in A$, there exists $b \in B$ such that $0 \in a \circledast b$. In particular, for every $x, y \in H, x \ll y$ if and only if $0 \in x \circledast y$. In such case, we call "«" the hyper order in $H$ (see [7]).

A hyper BCI-algebra $(H, \circledast, 0)$ is said to be ordered if for each $x, y, z \in H, x \ll y$ and $y \ll z$ imply $x \ll z$.

Example 1. [7] Let $H=\{0,1,2\}$. Define the hyperoperation " $\circledast$ " by the Cayley table shown below.

\begin{tabular}{c|ccc}
$\circledast$ & 0 & 1 & 2 \\
\hline 0 & $\{0,1\}$ & $\{0,1\}$ & $\{0,1\}$ \\
1 & $\{1\}$ & $\{0,1\}$ & $\{0,1\}$ \\
2 & $\{2\}$ & $\{1,2\}$ & $\{0,1,2\}$
\end{tabular}

Then by routine calculations, $(H, \circledast, 0)$ is a hyper BCI-algebra. Further, $H$ is ordered.

Let $\left(H_{1}, \circledast_{1}, 0_{1}\right)$ and $\left(H_{2}, \circledast_{2}, 0_{2}\right)$ be two hyper BCI-algebras. Consider a mapping $f: H_{1} \rightarrow H_{2}$. Then $f$ is said to be a homomorphism if $f\left(x \circledast_{1} y\right)=f(x) \circledast_{2} f(y)$, for all $x, y \in H_{1}$. If $f$ is a homomorphism and $f\left(0_{1}\right)=0_{2}$, then we call $f$ a hyper homomorphism. If $f$ is a homomorphism, one-to-one, and onto, we say that $f$ is an isomorphism and $\left(H_{1}, \circledast_{1}, 0_{1}\right)$ and $\left(H_{2}, \circledast_{2}, 0_{2}\right)$ are isomorphic, denoted by $H_{1} \cong H_{2}$ (see [6]).

Let $f: H_{1} \rightarrow H_{2}$ be a hyper homomorphism of hyper BCI-algebras. If $f$ is one to one (resp. onto) we say $f$ is a hyper monomorphism (resp. hyper epimorphism). If $f$ is a hyper homomorphism and a bijection, $f$ is said to be a hyper isomorphism, denoted by $H_{1} \cong_{\mathcal{H}} H_{2}$ (see [3]).

Throughout this study, we denote a hyper BCI-algebra $(H, \circledast, 0)$ by $H$, unless otherwise specified.

The following results generated previously give some of the properties of a hyper BCIalgebra.

Proposition 1. [7] In any hyper BCI-algebra H, the following hold:

(i) $x \ll 0$ implies $x=0$,

(ii) $0 \in x \circledast(x \circledast 0)$, 
(iii) $x \ll x \circledast 0$,

(iv) $0 \circledast(x \circledast y) \ll y \circledast x$,

(v) $A \ll A$,

(vi) $A \subseteq B$ implies $A \ll B$,

(vii) $A \ll\{0\}$ implies $A=\{0\}$,

(viii) $x \circledast 0 \ll\{y\}$ implies $x \ll y$,

(ix) $y \ll z$ implies $x \circledast z \ll x \circledast y$,

$(\mathrm{x}) x \circledast y=\{0\}$ implies $(x \circledast z) \circledast(y \circledast z)=\{0\}$ and $x \circledast z \ll y \circledast z$,

(xi) $A \circledast A=\{0\}$ implies $A$ is a singleton,

(xii) $A \circledast\{0\}=\{0\}$ implies $A=\{0\}$.

for all $x, y, z \in H$ and for all non-empty subsets $A$ and $B$ of $H$.

Theorem 1. [3] Let $f: H_{1} \rightarrow H_{2}$ be a hyper homomorphism. Then the following hold:

(i) If $x \ll y$, where $x, y \in H_{1}$, then $f(x) \ll f(y)$.

(ii) If $A, B \subseteq H_{1}$ such that $A \ll B$, then $f(A) \ll f(B)$.

\section{Zero Divisor Graph of a Hyper BCI-algebra}

Let $H$ be a hyper BCI-algebra and $A \subseteq H$. We will use the notation $L_{H}(A)$ to denote the set

$$
L_{H}(A):=\{x \in H \mid x \ll a, \forall a \in A\}=\{x \in H \mid 0 \in x \circledast a, \forall a \in A\} .
$$

If $A=\{a\}$, we write $L_{H}(\{a\})=L_{H}(a)$. For any $x \in H$, the set of zero divisors of $x$ is $Z_{x}=\left\{y \in H \mid L_{H}(\{x, y\})=\{0\}\right\}$.

Let $H$ be a finite hyper BCI-algebra. The zero divisor graph $\Gamma(H)$ of $H$ is the graph whose vertex set $V(\Gamma(H))=H$ and edge set $E(\Gamma(H))$ satisfying the following condition: for every distinct $x, y \in H, x y \in E(\Gamma(H))$ if and only if $L_{H}(\{x, y\})=\{0\}$ (equivalently, $x \in Z_{y}$ or $y \in Z_{x}$ ).

Although there are infinite hyper BCI-algebras, this paper only considers zero divisor graphs of finite hyper BCI-algebras.

Example 2. Consider the hyper BCI-algebra $H$ defined in Example 1.

Then $L_{H}(\{0,1\})=L_{H}(\{0,2\})=\{0\}$ and $L_{H}(\{1,2\})=\{0,1\}$. The zero divisors of $x \in H$ are $Z_{0}=\left\{y \in H \mid L_{H}(\{0, y\})=\{0\}\right\}=\{1,2\}$ and $Z_{1}=\{0\}=Z_{2}$. Thus, the zero divisor graph $\Gamma(H)$ of $H$ is given by the following figure: 


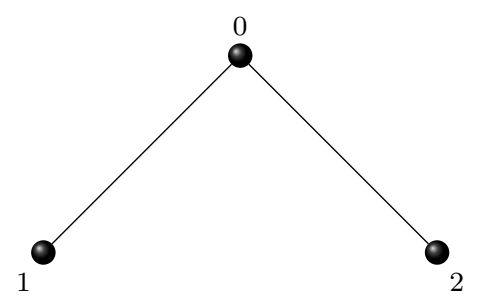

The next result gives some properties of the operator $L_{H}$.

Proposition 2. Let $A$ and $B$ be subsets of $H$. Then the following hold:

(i) $L_{H}(\varnothing)=H$

(ii) $L_{H}(\{0\})=\{0\}$

(iii) If $A \subseteq B$, then $L_{H}(B) \subseteq L_{H}(A)$.

(iv) $L_{H}(A)=\bigcap_{a \in A} L_{H}(\{a\})$

(v) If $x \in H$, then $x \in L_{H}(\{x\})$. Furthermore, $L_{H}(\{x\})=\{0\}$ if and only if $x=0$.

Proof.

(i) Suppose $L_{H}(\varnothing) \neq H$. Then $\exists h \in H$ such that $h \notin L_{H}(\varnothing)$; i.e., $\exists a \in \varnothing$ such that $a \nless h$, a contradiction. Therefore, $L_{H}(\varnothing)=H$.

(ii) By definition, $L_{H}(\{0\})=\{x \in H \mid x \ll 0\}=\{0\}$, by Proposition 1 .

(iii) Let $x \in L_{H}(B)$. Then $x \ll b, \forall b \in B$. Since $A \subseteq B, x \ll a, \forall a \in A$. Thus, $x \in L_{H}(A)$. Hence, $L_{H}(B) \subseteq L_{H}(A)$.

(iv) Follows from the definition of $L_{H}(A)$ :

$$
\begin{aligned}
L_{H}(A) & =\{x \in H \mid x \ll a, \forall a \in A\} \\
& =\left\{x \in H \mid x \in L_{H}(\{a\}), \forall a \in A\right\} \\
& =\bigcap_{a \in A} L_{H}(\{a\}) .
\end{aligned}
$$

(v) Let $x \in H$. By $\left(B_{3}\right), x \ll x$. Hence, $x \in L_{H}(\{x\})$. Furthermore, $x \in L_{H}(\{x\})=\{0\}$ implies $x=0$ and if $x=0$, then $L_{H}(\{x\})=L_{H}(\{0\})=\{0\}$ by (ii).

The zero divisor graph of a hyper BCI-algebra is not always connected: 
Example 3. Consider the hyper BCI-algebra $H$ with ' $\circledast$ ' defined by the following Cayley table:

\begin{tabular}{c|ccc}
$\circledast$ & 0 & 1 & 2 \\
\hline 0 & $\{0,1\}$ & $\{0,1\}$ & $\{2\}$ \\
1 & $\{1\}$ & $\{0,1\}$ & $\{2\}$ \\
2 & $\{2\}$ & $\{2\}$ & $\{0,1\}$
\end{tabular}

Then $L_{H}(\{0,1\})=\{0\} ; L_{H}(\{0,2\})=\varnothing=L_{H}(\{1,2\})$. Thus, the zero divisor graph $\Gamma(H)$ of $H$ is given below:
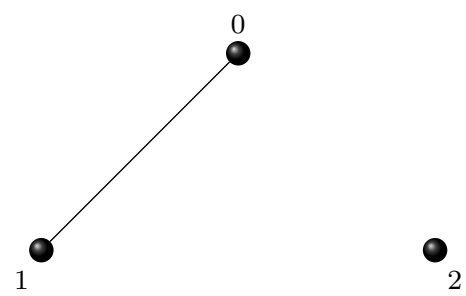

Example 4. Consider $H$ defined by the following Cayley table:

\begin{tabular}{c|cccc}
$\circledast$ & 0 & 1 & 2 & 3 \\
\hline 0 & $\{0\}$ & $\{0\}$ & $\{2\}$ & $\{2\}$ \\
1 & $\{1\}$ & $\{0\}$ & $\{2\}$ & $\{2\}$ \\
2 & $\{2\}$ & $\{2\}$ & $\{0\}$ & $\{0\}$ \\
3 & $\{3\}$ & $\{2\}$ & $\{1\}$ & $\{0,1\}$
\end{tabular}

Then $L_{H}(\{0,1\})=\{0\}, L_{H}(\{0,2\})=L_{H}(\{0,3\})=L_{H}(\{1,2\})=L_{H}(\{1,3\})=\varnothing$, and $L_{H}(\{2,3\})=\{2\}$. The zero divisor graph $\Gamma(H)$ of $H$ is given below

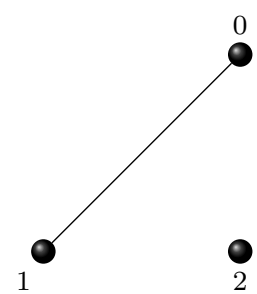

Proposition 3. Let $H$ be a hyper BCI-algebra with $|H| \geq 2$. Then

(i) $\operatorname{deg}_{\Gamma(H)}(0)=\left|\left\{x \in H \backslash\{0\}: 0 \in L_{H}(x)\right\}\right|=\Delta(\Gamma(H))$;

(ii) $\Gamma(H)=\bar{K}_{|H|}$ if and only if $0 \notin L_{H}(x)$ for all $x \in H \backslash\{0\}$; and

(iii) if $\Gamma(H) \neq \bar{K}_{|H|}$, then $\mathcal{I}(\Gamma(H))=\left\{x \in H \backslash\{0\}: 0 \notin L_{H}(x)\right\}$.

Proof. 
(i) Note that for any $x \in H \backslash\{0\}, 0 x \in E(\Gamma(H))$ if and only if $L_{H}(\{0, x\})=\{0\}$. Hence, by Proposition 2(ii), $0 x \in E(\Gamma(H))$ if and only if $0 \in L_{H}(x)$. Thus,

$$
\begin{aligned}
\operatorname{deg}_{\Gamma(H)} 0 & =|\{x \in H \backslash\{0\}: 0 x \in E(\Gamma(H))\}| \\
& =\left|\left\{x \in H \backslash\{0\}: 0 \in L_{H}(x)\right\}\right| .
\end{aligned}
$$

Let $x \in H \backslash\{0\}$ and let $y \in N_{\Gamma(H)}(x)$. Then $L_{H}(\{x, y\})=\{0\}$. By Proposition 2(ii) and 2(iv), it follows that $L_{H}(0, y)=\{0\}$, that is, $y \in N_{\Gamma(H)}(0)$. Thus,

$$
\operatorname{deg}_{\Gamma(H)}(x)=\left|N_{\Gamma(H)}(x)\right| \leq\left|N_{\Gamma(H)}(0)\right|=\operatorname{deg}_{\Gamma(H)}(0) .
$$

Since $x$ was arbitrarily chosen, it follows that $\Delta(\Gamma(H)) \mid=\operatorname{deg}_{\Gamma(H)}(0)$.

(ii) Suppose that $\Gamma(H)=\bar{K}_{|H|}$. That is, $\mathcal{I}(\Gamma(H))=V(\Gamma(H))$. This implies that $\operatorname{deg}_{\Gamma(H)} 0=0$. Hence, $0 x \notin E(\Gamma(H))$ for all $x \in H$. Thus, $0 \notin L_{H}(x)$ for all $x \in H \backslash\{0\}$.

For the converse, suppose that $0 \notin L_{H}(x)$ for all $x \in H \backslash\{0\}$. By (i), it follows that $\operatorname{deg}_{\Gamma(H)} 0=\Delta(\Gamma(H))=0$. Therefore, $\Gamma(H)=\bar{K}_{|H|}$.

(iii) Suppose that $\Gamma(H) \neq \bar{K}_{|H|}$. Then $\operatorname{deg}_{\Gamma(H)} 0=\Delta(\Gamma(H)) \neq 0$, i.e., $0 \notin \mathcal{I}(\Gamma(H))$. Let $x \in H \backslash\{0\}$. If $0 \notin L_{H}(x)$, then $L_{H}(x, y) \neq\{0\}$ for all $y \in H \backslash\{x\}$. Thus, $\operatorname{deg}_{\Gamma(H)}(x)=0$, i.e., $x \in \mathcal{I}(\Gamma(H))$. Conversely, if $x \in \mathcal{I}(\Gamma(H))$, then $0 x \notin E(\Gamma(H))$, i.e., $L_{H}(0, x) \neq\{0\}$. By Proposition 2(ii) and 2(iv), $0 \notin L_{H}(x)$. Therefore, $\mathcal{I}(\Gamma(H))=\left\{x \in H \backslash\{0\}: 0 \notin L_{H}(x)\right\}$.

Next, we give equivalent statements for connectedness of the zero divisor graph.

Proposition 4. Let $H$ be a hyper BCI-algebra with $|H| \geq 2$. Then the following are equivalent:

(i) $\Gamma(H)$ is connected.

(ii) $L_{H}(\{x, 0\})=\{0\}$ for all $x \in H \backslash\{0\}$.

(iii) $0 \in L_{H}(x)$ for all $x \in H \backslash\{0\}$.

(iv) $\Delta(\Gamma(H))=|H|-1$

(v) $\mathcal{I}(\Gamma(H))=\varnothing$

Proof.

(i) $\Leftrightarrow$ (ii) Suppose $L_{H}(\{x, 0\}) \neq\{0\}$ for some $x \in H$. Then $0 \notin L_{H}(x)$, by Proposition 2(ii) and 2(iv). Thus, $0 \notin L_{H}(\{x, y\})=L_{H}(\{x\}) \cap L_{H}(\{y\})$ for all $y \in H$. That is, for all $y \in H, x y \notin E(\Gamma(H))$. This implies that $\Gamma(H)$ is disconnected. For the converse, suppose that $L_{H}(\{x, 0\})=\{0\}$ for all $x \in H \backslash\{0\}$. Then $\operatorname{deg}_{\Gamma(H)}(0)=|H|-1$. Therefore, $\Gamma(H)$ is connected. 
(ii) $\Leftrightarrow$ (iii) This follows from Proposition 2(ii) and 2(iv).

(iii) $\Leftrightarrow$ (iv) This follows from Proposition 3(i).

(iv) $\Leftrightarrow(\mathrm{v})$ By Proposition 3(i), $\operatorname{deg}_{\Gamma(H)}(0)=|H|-1$. This implies that $0 x \in E(\Gamma(H))$ for each $x \in H \backslash\{0\}$. Hence, $\mathcal{I}(\Gamma(H))=\varnothing$. Conversely, suppose that $\mathcal{I}(\Gamma(H))=\varnothing$ and let $x \in H \backslash\{0\}$. Since $x \notin \mathcal{I}(\Gamma(H))$, there exists $y \in H \backslash\{x\}$ such that $L_{H}(x, y)=\{0\}$. Hence, $0 \in L_{H}(x)$ by Proposition 2(iv). By Proposition 3(i), it follows that $\operatorname{deg}_{\Gamma(H)}(0)=\Delta(\Gamma(H))=|H|-1$.

Remark 1. Let $H$ be a hyper BCI-algebra with $|H| \geq 2$. If $\Gamma(H)$ is connected, then

(i) $\operatorname{diam}(\Gamma(H))=2$;

(ii) $\operatorname{deg}_{\Gamma(H)} 0=|H|-1=\Delta(\Gamma(H))$.

Proposition 5. Let $H$ be a hyper BCI-algebra such that $L_{H}\{x, 0\} \neq \varnothing$ for all $x \in H$. Then $L_{H}\{x, 0\}=\{0\}$.

Proof. Suppose that $L_{H}\{x, 0\} \neq \varnothing$ for all $x \in H$. Then there exists $y \in L_{H}\{x, 0\}$. Note that $y \in L_{H}\{0\}=\{0\}$ means that $y=0$. It follows from Proposition 2(ii) and 2(iv) that $L_{H}\{x, 0\}=\{0\}$.

Remark 2. Let $H$ be a hyper BCI-algebra such that $L_{H}\{x, 0\} \neq \varnothing$ for all $x \in H$. Then $0 x \in E(\Gamma(H)) \forall x \in H \backslash\{0\}$.

Proposition 6. If $|H|>3$, then $\Gamma(H)$ is neither a cycle nor a path.

Proof. Case 1. $\exists x \in H \backslash\{0\}$ such that $0 \notin L_{H}(x)$. Then $\Gamma(H)$ is disconnected, and the result follows. Case 2. $0 \in L_{H}(x) \forall x \in H$. Then $0 x \in E(\Gamma(H)) \forall x \in H \backslash\{0\}$. Evidently, $\Gamma(H)$ is neither a cycle nor a path.

Corollary 1. If a graph $G$ is a cycle or a path of order $n \geq 4$, then there is no hyper $B C I$-algebra $H$ such that $\Gamma(H) \cong G$.

Proof. Immediate from Proposition 6.

Theorem 2. Let $H$ be a hyper BCI-algebra with $|H| \geq 2$. Then $G=\Gamma(H)$ cannot have two nontrivial components; that is, $G$ can only have at most one non-trivial component.

Proof. If $G$ is connected, then we are done. Suppose that $G$ is disconnected. Suppose further that $G$ has two distinct non-trivial components, say $G_{1}$ and $G_{2}$. Let $G_{3}$ be a component of $G$ with $0 \in V\left(G_{3}\right)\left(G_{3}\right.$ may be $G_{1}$ or $\left.G_{2}\right)$. If $G_{3}$ is different from $G_{1}$, then $0 \notin L_{H}(x)$ for all $x \in V\left(G_{1}\right)$. Similarly, if $G_{3}$ is not $G_{2}$, then $0 \notin L_{H}(y)$ for all $y \in V\left(G_{2}\right)$. Hence, by Proposition $4, G_{1}$ or $G_{2}$ is the trivial graph, a contradiction. 
Proposition 7. Let $H$ be an ordered hyper BCI-algebra. Then the following hold:

(i) For any subset $A$ of $H, L_{H}\left(L_{H}(A)\right) \subseteq L_{H}(A)$.

(ii) For any $a, b \in H$, if $a \ll b$, then $L_{H}(\{a\}) \subseteq L_{H}(\{b\})$ and $Z_{b} \subseteq Z_{a}$.

Proof.

(i) Let $x \in L_{H}\left(L_{H}(A)\right)$. Then $x \ll b$ for all $b \in L_{H}(A)$. Since $b \ll a$ for all $a \in A$ and $H$ is ordered, it follows that $x \ll a$ for all $a \in A$. Thus, $x \in L_{H}(A)$ and the result follows.

(ii) Suppose $x \in L_{H}(\{a\})$. Then $x \ll a$. Since $H$ is ordered and $a \ll b, x \ll b$. That is, $x \in L_{H}(\{b\})$. Hence, $L_{H}(\{a\}) \subseteq L_{H}(\{b\})$. Now, suppose $x \in Z_{b}$. Then $L_{H}(\{b, x\})=\{0\}$. Since $L_{H}(\{a, x\}) \subseteq L_{H}(\{b, x\})$, we have $L_{H}(\{a, x\})=\{0\}$. This means that $x \in Z_{a}$. Thus, $Z_{b} \subseteq Z_{a}$.

Proposition 8. Let $H$ be a hyper BCI-algebra. Then

(i) $\operatorname{deg}_{\Gamma(H)} x=\left|Z_{x}\right|$ for all nonzero $x \in H$.

(ii) $y \in Z_{x}$ if and only if $x \in Z_{y}$ for all $x, y \in H$.

Proof. Let $x, y \in H$.

(i) if $x \neq 0$, then

$$
\begin{aligned}
\left|Z_{x}\right| & =\left|\left\{y \in H \backslash\{x\}: L_{H}(\{x, y\})=\{0\}\right\}\right| \\
& =|\{y \in H: x y \in E(\Gamma(H))\}| \\
& =\operatorname{deg}_{\Gamma(H)} x .
\end{aligned}
$$

(ii) $y \in Z_{x}$ means that $L_{H}(\{x, y\})=\{0\}$, which further means that $x \in Z_{y}$.

Lemma 1. Let $f: H_{1} \rightarrow H_{2}$ be a hyper monomorphism of hyper BCI-algebras. Then for any $x, y \in H_{1}, x \ll y$ if and only if $f(x) \ll f(y)$.

Proof. The sufficiency part is done by Theorem 1(i). Now, suppose $f(x) \ll f(y)$. Then $0_{2} \in f(x) \circledast_{2} f(y)=f\left(x \circledast_{1} y\right)$. Thus, $0_{1}=f^{-1}\left(0_{2}\right) \in f^{-1} f\left(x \circledast_{1} y\right)=x \circledast_{1} y$. Hence, $x \ll y$.

Proposition 9. Let $f: H_{1} \rightarrow H_{2}$ be a hyper monomorphism of hyper BCI-algebras. Then $L_{H_{2}}(f(A))=f\left(L_{H_{1}}(A)\right)$ where $A \subseteq H_{1}$. 
Proof. Let $f: H_{1} \rightarrow H_{2}$ be a hyper monomorphism. Let $A \subseteq H_{1}$.

$$
\begin{aligned}
y \in f\left(L_{H_{1}}(A)\right) & \Longleftrightarrow f^{-1}(y) \in L_{H_{1}}(A) \\
& \Longleftrightarrow f^{-1}(y) \ll a \text { for all } a \in A \\
& \Longleftrightarrow y \ll f(a) \text { for all } a \in A \text {, by Lemma } 1 \\
& \Longleftrightarrow y \in L_{H_{2}}(f(A))
\end{aligned}
$$

Therefore, $L_{H_{2}}(f(A))=f\left(L_{H_{1}}(A)\right)$.

Theorem 3. Let $H_{1}$ and $H_{2}$ be hyper BCI-algebras. If $H_{1} \cong_{\mathcal{H}} H_{2}$, then $\Gamma\left(H_{1}\right) \cong \Gamma\left(H_{2}\right)$.

Proof. Suppose $H_{1} \cong_{\mathcal{H}} H_{2}$, say $f: H_{1} \rightarrow H_{2}$ is a hyper isomorphism. Since $V\left(\Gamma\left(H_{1}\right)\right)=H_{1}$ and $V\left(\Gamma\left(H_{2}\right)\right)=H_{2}$, there exists a one-to-one correspondence between the vertex sets. Note that for any distinct elements $x, y \in H_{1}, x y \in E\left(\Gamma\left(H_{1}\right)\right)$ if and only if $L_{H_{1}}(\{x, y\})=\{0\}$. By Proposition 9, xy $\in E\left(\Gamma\left(H_{1}\right)\right)$ if and only if $L_{H_{2}}(\{f(x), f(y)\})=\{0\}$. Thus, $x y \in E\left(\Gamma\left(H_{1}\right)\right)$ if and only if $f(x) f(y) \in E\left(\Gamma\left(H_{2}\right)\right)$. Consequently, $\Gamma\left(H_{1}\right) \cong \Gamma\left(H_{2}\right)$.

\subsection{On zero divisor graphs involving hyperatoms}

Definition 1. An element a of a hyper BCI-algebra $H$ is called a hyperatom if for each $x \in H, x \ll a$ implies $x=0$ or $x=a$.

Denote by $A(H)$ the set of all hyperatoms of $H$, and by $A^{*}(H)$ the set of all nonzero hyperatoms of $H$; i.e., $A^{*}(H)=A(H) \backslash\{0\}$. Obviously, $0 \in A(H)$.

Definition 2. A hyper BCI-algebra $H$ is said to be hyperatomic if each element of $H$ is a hyperatom, that is, $A(H)=H$.

Remark 3. A hyper BCI-algebra $H$ is hyperatomic if and only if $L_{H}(x)=\{x\}$ or $L_{H}(x)=$ $\{0, x\}$ for each $x \in H$.

Remark 4. A hyperatomic hyper BCI-algebra is ordered.

Proof. Suppose $H$ is a hyperatomic hyper BCI-algebra. Let $x, y, z \in H$ such that $x \ll y$ and $y \ll z$. Then by Remark $3, L_{H}(z)=\{z\}$ or $L_{H}(z)=\{0, z\}$. Thus, $y \ll z$ implies that $y=0$ or $y=z$. If $y=0$, then $x \ll 0$ since $x \ll y$. By Proposition $1, x=0$. Since $0 \ll z$ and $x=0$, we have $x \ll z$. If $y=z$, then the assumption $x \ll y$ implies that $x \ll z$. Hence, $H$ is ordered.

Example 5. Consider the hyper BCI-algebra defined by the Cayley table:

\begin{tabular}{c|ccc}
$\circledast$ & 0 & 1 & 2 \\
\hline 0 & $\{0\}$ & $\{0,1\}$ & $\{0,1\}$ \\
1 & $\{1\}$ & $\{0,1\}$ & $\{1\}$ \\
2 & $\{2\}$ & $\{2\}$ & $\{0,1,2\}$
\end{tabular}


$\mathrm{H}$ is hyperatomic since all its elements are hyperatoms.

Example 6. The hyper BCI-algebra $H$ in Example 1 is not hyperatomic since 2 is not a hyperatom of $\mathrm{H}: \exists x=1 \in H$ with $1 \ll 2$ but $x=1 \neq 0$ and $x=1 \neq 2$. However, the hyper BCI-algebra $H$ in Example 3 is hyperatomic.

Proposition 10. Let $H$ be a hyper BCI-algebra such that $|H| \geq 2$. If $x$ and $y$ are distinct nonzero hyperatoms of $H$, then $L_{H}(\{x, y\})=\{0\}$ or $\varnothing$.

Proof. The result depends on whether or not $0 \in L_{H}(x)$ for all $x \in H$. If $0 \notin L_{H}(x)$, then $L_{H}(x)=\{x\}$. Hence, $L_{H}(\{x, y\})=\varnothing$. If $0 \in L_{H}(\{x\})$, then $L_{H}(\{x\})=\{0, x\}$. Since $L_{H}(\{y\})=\{0\}$ or $\{0, y\}$ by Remark 3 , we have $L_{H}(\{x, y\})=\{0\}$ or $\varnothing$.

We have the following characterization for a complete graph:

Proposition 11. Let $H$ be a hyper BCI-algebra such that $|H| \geq 2$. Then $\Gamma(H)$ is a complete graph if and only if $\Gamma(H)$ is connected and $H$ is hyperatomic.

Proof. If $\Gamma(H)$ is disconnected, then $\Gamma(H)$ is not a complete graph, and we are done. Assume that $\Gamma(H)$ is connected. By Proposition 4, $0 \in L_{H}(x)$ for all $x \in H$. Since $x \in L_{H}\{x\}$, we now have $0, x \in L_{H}(x)$. If $H$ is not hyperatomic, then there exists $z \in H \backslash\{0\}$ such that $y \ll z$ with $y \notin\{0, z\}$. Since $y \in L_{H}\{y\}, y \in L_{H}\{y, z\}$. This means that $L_{H}\{y, z\} \neq\{0\}$, implying that $y z \notin E(\Gamma(H))$. Therefore, $\Gamma(H)$ is not complete.

Conversely, suppose $\Gamma(H)$ is connected and $H$ is hyperatomic. Then by Remark 3 , $L_{H}\{x\}=\{0, x\}$ for all $x \in H \backslash\{0\}$. Thus, for any distinct nonzero elements $x, y$ of $H=V(\Gamma(H)), L_{H}\{x\} \cap L_{H}\{y\}=\{0\}$, that is, $x y \in E(\Gamma(H))$. Consequently, $\Gamma(H)$ is a complete graph.

Example 7. The hyper BCI-algebra in Example 5 has a complete zero divisor graph:

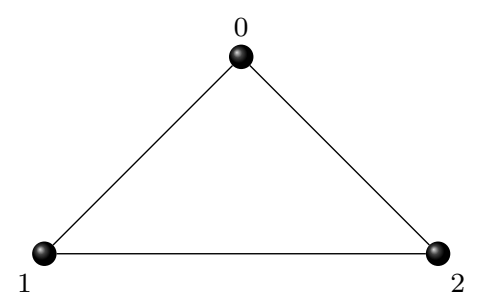

Remark 5. Given an ordered hyper BCI-algebra $H$, it is not always true that there exists $a \in A^{*}(H)=A(H) \backslash\{0\}$ such that $a \ll x$ for all $x \in H \backslash\{0\}$.

Example 8. Consider the hyper BCI-algebra $H$ defined in Example 3. $H$ is hyperatomic and hence, ordered and $A^{*}(H)=\{1,2\}$. Notice that neither $1 \ll x$ nor $2 \ll x$ for all $x \in H \backslash\{0\}$. But for each $x \in H \backslash\{0\}$, there exists $a \in A^{*}(H)$ such that $a \ll x$.

Theorem 4. Let $H$ be an ordered hyper BCI-algebra with $|H| \geq 2$. Then the following hold: 
(i) For each $x \in H$, there is $a_{x} \in A^{*}(H)$ such that $a_{x} \ll x$. In particular, $A^{*}(H) \neq \varnothing$.

(ii) There exists $a \in H \backslash\{0\}$ such that $a \ll x$ for all $x \in H \backslash\{0\}$ if and only if $\left|A^{*}(H)\right|=1$ (that is, $A^{*}(H)=\{a\}$ ).

Proof.

(i) Let $x \in H \backslash\{0\}$. If $x$ is a hyperatom, then take $a_{x}=x$. If $x$ is not a hyperatom, there exists $x_{1} \in H \backslash\{0, x\}$ such that $x_{1} \ll x$. Again, if $x_{1}$ is a hyperatom, then take $a_{x}=x_{1}$. Otherwise, there exists $x_{2} \in H \backslash\left\{0, x, x_{1}\right\}$ such that $x_{2} \ll x_{1} \ll x$. Since $H$ is finite, continuing in this fashion yields a terminal point $x_{n} \in H \backslash\left\{0, x, x_{1}, \ldots, x_{n-1}\right\}$ with $x_{n} \ll x_{n-1} \ll \cdots \ll x_{2} \ll x_{1} \ll x$ such that only $z=0$ (provided $0 \in L_{H}\{x\}$ ) or $z=x_{n}$ satisfies $z \ll x$. This implies that $a_{x}=x_{n} \in A^{*}(H)$ and $a_{x} \ll x$.

(ii) Suppose that there exists $a \in H \backslash\{0\}$ such that $a \ll x$ for all $x \in H \backslash\{0\}$. Choose any $b \in A^{*}(H)$. Then $a \ll b$. Since $b \in A(H)$ and $a \neq 0$, it follows that $a=b$. Thus $a \in A^{*}(H)$. Since $b$ was arbitrarily chosen, we have $A^{*}(H)=\{a\}$. Conversely, suppose that $\left|A^{*}(H)\right|=1$, say $A^{*}(H)=\{a\}$. Let $x \in H \backslash\{0\}$. Then by (i), $a \ll x$.

Example 9. Consider the ordered hyper BCI-algebra $H$ defined in Example 1. Note that 1 is the only nonzero hyperatom of $H$ and the zero divisor graph $\Gamma(H)$ of $H$ is a star.

As a generalization of Example 9, we have the following theorem.

Theorem 5. Let $H$ be an ordered hyper BCI-algebra with $|H| \geq 2$. Then $\Gamma(H)$ is a star if and only if $\Gamma(H)$ is connected and $\left|A^{*}(H)\right|=1$.

Proof. Suppose that $\Gamma(H)$ is a star. Then $\Gamma(H)$ is connected. If $|H|=2$, then clearly, $\left|A^{*}(H)\right|=1$. Suppose that $|H| \geq 3$. By Proposition 3(i), 0 is the central vertex of $\Gamma(H)$. Suppose further that $\left|A^{*}(H)\right| \geq 2$, say $a, b \in A^{*}(H)$ with $a \neq b$. Since $0 a, 0 b \in E(\Gamma(H))$, $0 \in L_{H}(a) \cap L_{H}(b)$. By Proposition $10, a b \in E(\Gamma(H))$. This implies that $\Gamma(H)$ is not a star, a contradiction. Therefore, $\left|A^{*}(H)\right|=1$.

Conversely, suppose that $\Gamma(H)$ is connected and $\left|A^{*}(H)\right|=1$, say $A^{*}(H)=\{a\}$. If $|H|=2$, then $\Gamma(H)=P_{2}$, a star. Suppose that $|H| \geq 3$ and let $y, z \in H \backslash\{0\}$. By Theorem 4(ii), $a \ll y$ and $a \ll z$. That is, $a \in L_{H}(\{y, z\})$. This implies that $y z \notin E(\Gamma(H))$. Since $\Gamma(H)$ is connected, by Proposition $4,0 x \in E(\Gamma(H))$ for all $x \in H \backslash\{0\}$. Consequently, $\Gamma(H)$ is a star.

\section{Acknowledgements}

This research is funded by the Philippine Department of Science and TechnologyAccelerated Science and Technology Human Resource Development Program (DOSTASTHRDP) and Mindanao State University-Iligan Institute of Technology. 


\section{References}

[1] Beck, I., Coloring of Commutative Rings, Journal of Algebra, 116 (1988), 208-226.

[2] DeMeyer, F.R., McKenzie, T. and Schneider, K., The Zero-Divisor Graph of a Commutative Semigroup, Semigroup Forum, 65 (2002), 206-214.

[3] Flores, G.B.C. and Petalcorin, G.C., Some Hyper Isomorphism Theorems of Hyper BCI-algebras, Journal of Algebra and Applied Mathematics, 13 (2015), 15-31.

[4] Harary, F., Graph Theory, Addison-Wesley Publishing company, Inc.,USA, 1969.

[5] Jun, Y.B. and Lee, K.J., Graphs Based on BCK/BCI-Algebras, International Journal of Mathematics and Mathematical Sciences, 2011 (2011), 1-8.

[6] Nisar, F., Tariq, R.S. and Bhatti, S.A., Fuzzy Ideals in Hyper BCI-Algebras, World Applied Science Journal, 12(2012), 1771-1777.

[7] Xin, X.L., Hyper BCI-algebras, Discuss Math. Soc., 26(2006), 5-19. 